\title{
On example of circular arc approximation by quadratic minimal splines
}

\author{
A.A. Makarov \\ Date of Receiving : $\quad 04.04 .2018$ \\ Date of Revision : $\quad 10.12 .2018$ \\ Date of Acceptance : $\quad 24.12 .2018$
}

\begin{abstract}
The paper considers the quadratic minimal splines and local spline approximation scheme with explicit dual approximation functionals. A simple case of circular arc (quarter of a circle) approximation is regarded. Numerical results of spline approximation are presented.
\end{abstract}

\section{Introduction}

Circles and circular arcs are widely used in computer-aided design systems. It is known, that it is not possible to represent a circle with a polynomial curve in explicit form, but it can be represented, for example, by a quadratic rational spline. A circular arc does not have a parametric polynomial representation, but it can be represented by using a quadratic rational curve, e. g. Bézier or NURBS. There are a lot of papers devoted to circular arc approximation (see $[1,2,3]$ and references therein). Nevertheless the construction of a circular arc approximation with the highest possible accuracy is a very important issue.

This note is motivated by the paper [3] and the reference on the paper [4] given there. Although it is shown [5] that spline curves can approximate with higher order than spline functions, we start with approximation by spline functions constructed from approximation relations by using a complete chain of vectors and a generating vector function $\boldsymbol{\varphi}$ (see, e.g., [6]); such splines have a minimal support and a maximum degree of smoothness $\left(B_{\varphi}\right.$-splines).

Quadratic polynomial $B$-splines are the partial case of $B_{\varphi}$-splines generated by the vector function $\boldsymbol{\varphi}=\left(1, t, t^{2}\right)^{T}$ (see [7]). The establishment of conditions for the sign definiteness of minimal splines is important for practical application in geometric design problems. For example, the quadratic hyperbolic minimal splines generated by vector function $\varphi(t)=(1, \sinh t, \cosh t)^{T}$ are positive without any

2010 Mathematics Subject Classification. 41A05, 41A15, 65D07.

Key words and phrases. minimal spline, $B$-spline, dual approximation functional, circular arc approximation.

Communicated by: S.K. Kaushik 